\title{
Comentario al trabajo sobre la aplicación del método sicoprofiláctico en Medellín (*)
}

\author{
Doctor M. A. Fernández-Bastidas (;)
}

\section{Clases teóricas y tipo de pacientes}

El trabajo presentado al "Primer Simposio Colombiano sobre Parto Indoloro" celebrado en Bogotá, por los doctores G. Isaza Mejía, J. Muñoz Moreno y L. Rodríguez, es un estudio metódico que da a cabalidad una idea precisa sobre los resultados obtenidos por los autores a través de dos años y medio de su aplicación en la clientela privada en la ciudad de Medellín (Colombia).

El plan de conferencias seguido calca punto por punto el plan esbozado por F. Lamaze y colaboradores de la Clínica de los Metalúrgicos de París; salvo lo referente a la primera conferencia, en la cual, a manera de introducción, enfoca el aspecto religioso con la lectura de la carta del Papa Pío XII, factor que se ha tenido también muy en cuenta en nuestras pacientes de la Clínica de Palermo, de Bogotá, por parecer ineludible tratar el punto (sentencia bíblica) con pacientes de gran sentido religioso como son la gran mayoría de las mujeres colombianas.

El tipo de pacientes, a juzgar por anotación de los autores antioqueños, es algo heterogéneo (pacientes sin discriminación de edad de embarazo, capacidad intelectual, posición económica, etc.), rio obstante que la selección en la agrupación, según ellos mismos, son "factores tan importantes en el éxito".

Sobre el número total de casos entrenados (260) solamente un $66 \%$ fue atondido por el método, y de éstos solamente fueron anotados los resultados correspondientes a 52 .

(*) Comentario hecho por el autor para el Primer Simposio Colombiano de Sicoprofilaxia, leído durante las sesiones de éste.

(**) Secretario del Equipo Médico de Estudios Sicofísicos de Analgesia Obstétrica de Bogotá (EMESFAO). Secretario General del Primer Simposio Colombiano de Sicoprofilaxia Obstétrica (febroro de 1959). 
Las razones aducidas para no haber sido atendido por el método la totalidad de las pacientes preparadas, apareciendo como atendidas por otros métodos, parece deberse a una falla en cuanto a la falta de información y experiencia de los obstetras que deberían atender las pacientes entrenadas por los doctores Isaza Meja, Muñoz Moreno y Rodriguez; falla que parece ha tendido a corregirse, habiendo sido atendidos por ellos los tiltimos casos presentados.

Otro factor adverso, digno dé tenerse en cuenta para en un futuro mejorar los resultados, es la libre escogencia de clínica por parte de las pacientes donde deben ser atendidas, pues uno de los puntos sobre los cuales más insisten, tanto los autores rusos como franceses, es la ventaja de centralizar en una sola entidad hospitalaria, no solamente la atención de los cascis con personal integramente ilustrado al respecto, sino que la misma instrucción debé llevarse a cabo en lial lugar, por obtener con las visitas periódicas para recibir ia insiruccion, una serie de excitantes favorables en el momento que la enferma ingresa a tener su parto.

For bitimo, réstanos comentar la ausencia en el programa de clases teóricas, de una conferencia dedicada a los maridos, o la aclaración, si a todas las instrucciones teóricas asisten las parejas. Fues si bien no todos los maridos, por sus ocupaciones o por no quererle dar la importancia que se merece el estado de su consorte, o la perspectiva de un éxito con el método, son algo remisos a concurrir en las primeras oportunidades; tal exigencia podria serles insinuada a las pacientes para que por su intermedio se lograra una concurrencia cada vez mayor de maridos al curso; logrando en esta forma una mayor cooperación en el hogar, benéfica para el condicionamiento de la señora durante el embarazo, y más aún como ayuda efectiva en el momento del parto.

En cuanto a las clases teóricas, debemos comentar igualmente la importancia de una mayor elasticidad en cuanto a las conferencias y conferenciantes se refiere, a través de los distintos cursos de entrenamiento; pues si bien se halla plenamente justificado que temas relacionados esencialmente con aspectos puramente sicológicos o pediátricos deban ser presentados siempre por $e_{i}$ mismo conferenciante suficientemente informado sobre el tema; el resto del equipo formado por especialistas en obstetricia y ginecología logra una mayor cohesión y obtiene una vinculación más efectiva, firme y conjunta, al evitar dictar el mismo confereciante siempre la misma conferencia. 
En cuanto a la importancia de las clases teóricas en general, parécenos de interés hacer algunas observaciones complementarias al punto que nos toca comentar. A pesar de la opinión de L. Chertok, quien afirma que los conceptos teóricos sobre' los cuales se funda el método sicoprofiláctico, no están aún hoy día tan suficientemente aclarados como la hipno-sugestión, que reposa en la efectividad de la sugestión post-hipnótica; debemos considerar tres elementos esenciales de acción analgésica en el método sicoprofiláctico:

a) El eliemento didáctico, que tiene que ver directamente con las clases teóricas, en su manera de organizarlas y hacerlas accesibles a la mentalidad de las alumnas;

b) Los elementos fisioterápicos, y

c) Los elementos sicoterápicos. Estos dos últimos, al decir de Jeanson, no siendo considerados separadamente, sino como un todo: el elemento sicofísico.

Además del extraordinario poder de señalización cortical que constituye el lenguaje, el aspecto didáctico, en cuya falla muy posiblemente puede ir comprometido el éxito de determinado plan de conferencias, actúa como elemento informativo, familiarizando a la embarazada con los hechos anatomo-fisiológicos, cuyo conjunto constituye el acto del parto; anulanado en tal forma la incógnita que en una gran parte de los casos es la causa del temor a éste.

Hablando en lenguaje sico-analítico, podemos citar el concepto de Donován y Landisberg, quienes consideran que el elemento didáctico opera sustituyendo "fantasmas" por hechos; con la salvedad que tal sustitución no podrá lograrse a cabalidad sino En estados exentos de grados avanzados de angustia, casos en los cuales el obstetra deberá estar esesorado de un siquiatra que trate cada caso de manera apropiada. Así la angustia, considerada como un grado más avanzado del temor, no es susceptible de ser anulada únicamente por la sicoprofilaxia, pues ésta al decir de Quijada, no obrará allí de manera efectiva.

Kubie opina, a propósito de la educación pre-natal: "La gravedad del choque causada por el dolor debida a una amenaza inminente o a una realidad corriente, puede ser disminuida si la angustia y los fantasmas que la acompañan (tanto conscientes como inconscientes) pueden ser disminuídos"; de esta manera, la angustia llegaría en parte a ser anulada "por una educación que muestre de una manera viva y franca la naturaleza del cuerpo, su 
Iuncionamiento normal, su capacidad de adaptación, así como su vulnerabilidad". Disminuyendo el misterio del cuerpo, se pueden generalmente disminuir los terrores que trae consigo el parto.

Chertok dice: "La enseñanza es igualmente importante tanto en el plano social, elevando el nivel de instrucción, como en el plano individual, en que la persona ve aumentadas sus adquisiciones y posibilidades en favor del reforzamiento del yo"; y agrega: "El elemento didáctico hace entrar la gestante en una relación interpersonal con el preparador y los miembros de su grupo, lo cual constituye un factor sicoterápico". Sinembargo, según lo anotaba Konstantinov en el Congreso de Kiev, el conocimiento solo no es suficiente para producir analgasia, puesto que las mujeres-médicos y las parteras, a pesar de los conocimientos médicos propios de su profesión, hacen en su gran mayoría partos dolorosos.

Kubie asigna igualmente límites a la pedagogía al decir que: "hay un límite específico a la disminución de la angustia por la sola educación; tal límite se encontraría en las fronteras de las neurosis, allí donde las fuentes inconscientes y deformadas de la angustia entran en escena", "los procesos inconscientes sicológicois, no son accesibles a la educación, así como no lo son a las recompensas o castigos, a los argumentos razonados, a las exhortaciones, o a las amenazas".

Siendo el elemento didáctico un procedimiento típico de sicoterapia, fácilmente podemos admitir la existencia de un fenómeno de transferencia entre la paciente y el obstetra, transferencia de la imagen paterna en la mayoría de los casos; siendo, al decir de Seguin, "el primer deber del sicoterapeuta, comprender la naturaleza de la relación emocional y reconocer la o las figuras, que está representando en ese momento para la paciente".

\section{Clases prácticas}

En lo relativo a las clases prácticas, el equipo del doctor Isaza Mejía, de Miedellín, insiste sobre la importancia del entrenamiento físico para llegar al parto en buenas condiciones, en la sencillez de su práctica y en el poco tiempo que requiere diariamente.

Los ejercicios, en número de cinco, son explicados con la mayor claridad, valiéndose de ilustraciones y diagramas. La parte reiacionada con respiración, iniciada con una explicación contenida en el libro del doctor Isaza sobre la gran importancia de la oxigenación, es puesta en práctica con el clásico ejercicio de respiración consciente, preconizada por la escuela de los Metalúrgi- 
cos de Paris, y que simulando soplar una vela le hace tomar conciencia a la embarazada de tal acto de manera gradual.

La percepción de la contracción uterina que comprende el segundo ejercicio practicado de acuerdo con las normas clásicas, es acompañado acertadamente de la advertencia de que tal contracción, percibida durante el embarazo, es perfectamente indolora.

La adaptación respiratoria durante la contracción uterina, denominada por el doctor Isaza "respiración uniforme", imita en un todo el tipo de respiración adoptada po: F. Lamaze y Vellay, procuranado una mayor frecuencia con menor amplitud en el jiempo correspondiente a la contracción uterina, e insistiendo en que se debe practicar con la boca abierta y no por la nariz. En lo relacionado con tal tipo de respiración debemos anotar la relativa frecuencia dé sintomas debidos a cambios ácido-básicos indeseables en la sangre de las pacientes, producidos por la hiperventilación, tales como la āparición de adormecimiento en cara y manos, y observado en cinco de los casos presentados en nuestra rrimera comunicación; además del resecamiento de la mucosa oral y faringea después de la prolongada práctica de tal modalidad de respiración, motivo por el cual nos vimos precisados a sustituírla por respiración por nariz, siguiendo la técnica dada por ia escuela rusa.

La respiración jadeante ocupa capitulo especial en el plan de clases prácticas; únicamente cabe notar, la mucha importancia dada al "golpe que el diafragma produce en cada contracción sobre el útero", dando importancia secundaria al frenado cortical obtenido mediante tal práctica.

El capitulo relativo al pujo es, sin duda, la parte más claramente presentada en el folleto destinado a ser entregado a las señoras.

Por último, el extenso y muy claro capítulo relativo al control neuro-muscular, mecanismo y objeto de la respiración y relajación muscular; complementa suficientemente el ya señalado foIleto de instrucción práctica.

\section{Apoyo del trabajo y condiciones de la clínica}

Por todos los autores es admitida como condición esencial para obtener éxitos con la preparación sicoprofiláctica para el parto, que a más del entrenamiento apropiado, debe la embarazada estar suficientemente vigilada y apoyada en tal momento, para 10 cual ha recibido útil y suficiente instrucción durante el embarazo. 
Los casos observados tuvieron apoyo efectivo por "una enfermera preparada en el método", a fin de evitar contratiempos y errores de personal inexperto. Igualmente afirman los autores que "el obstetra vigiló desde un principio la revolución del trabajo" desde 5 centímetros hasta dilatación completa. Pero de ios 260 casos solamente 172 , o sea el $66 \%$, fueron ralmente atendidas y vigiladas con tales normas. ¿Cuál fua el motivo para que en 88 casos prácticamente se hubiera perdido la preparación de las pacientes? Sin duda el hecho de tratarze de señoras cuyo obstetra no tenía una información suficiente, o desconocía las bases y por ende los resultados que hubiera podido obtener valiéndose de tal recurso.

Para obviar tal problema ya señalamos anteriormente el requisito de presentar cada paciente en el momento de matricularse al curso, una constancia escrita, en la cual el obstetra, que más tarde deberá atenderla, se comprometa a vigilarla desde su ingreso a la clínica, sin interferir su actitud sicoprofiláctica, prestánciole ei apoyo que requiera. De otra manera, una paciente con preparación buena puede fácilmente sentirse defraudada del método, única y exclusivamente por falla del elemento médico.

En lo relativo a la clínica consideramos como un error el hecho de dejar "la elección de ésta al gusto de la señora", pues las ventajas de la centralización de este tipo de pacientes en una sola entidad hospitalaria a más de faciliar la vigilancia de los casos, condiciona favorablemente a las pacientes para que en el momento de internarse ya tengan suficiente confianza en el personal y el medio en el cual van a permanecer durante el trabajo.

Lo ideal en el método sicoprofiláctico y su aplicación, es que el sitio de instrucción funcione anexo a la clínica donde la paciente más tarde debe ser atendida. En tal forma funciona el hospital de los Metalúrgicos de París, y en Colombia varios de los equipos han encontrado una ventaja notable con tal organización.

Es tan importante el factor ambiental, que si bien los mismos autores franceses reconocen su influencia favorable a juzgar por sus publicaciones, reconocen no haber llegado aún a tener un centro hospitalario ideal, que consulte todas las necesidades y requerimientos de tranquilidad y confort para la atención óptima, en lo relacionado con la clínica, de pacientes entrenadas según el método.

C. Jeanson describe la clínica ideal en la siguiente forma: "Tal maternidad debe tener un acceso fácil, enmarcado por jardines, debe ser aireada y absolutamente tranquila. Nada que pue- 
da dar un aspecto frío o austero, es decir, nada que pueda en un momento producir inquietud. Un vestíbulo con flores y grandes vitrales, etc.", y al hablar del personal médico y de enfermería dice: "Cada uno de sus elementos debe siempre contribuir a la atmósfera sicológica indispensable, teniendo cuatro cualidades que nos parecen esenciales: amabilidad, comprensión, tranquilidad y dulzura".

\section{Datos estadísticos y clasificación de resultados}

Los autores del trabajo de Medellín dividen los casos presentados en dos épocas: la primera de septiembre de 1956 a diciembre del 57 ( 15 meises), y la segunda todo el año de 1958.

De los casos analizados en la primera época, 75 fueron casos de parto que no requirieron anestesia y 15 en los cuales fue necesario emplearla, aunque se dice que sí obtuvieron beneficios con el entrenamiento.

Entre los casos de la segunda época, con mejor orientación a nuestro modo de ver, 52 pacientes fueron atendidas; su clasificación fue diferente a la adoptada en la primera serie, y a diferencia de aquélla se aceptan cuatro en lugar de tres numerales, graduados de 2 a 5 ; siendo tenidos como éxitos los calificados como 3 , 4 y 5 ; pudiendo hacerse la objeción que la calificación 3, correspondiente a casos con "tendencia al descontrol, pero reparable, dolor đébil persistente, sin necesidad de anestesia", son éxitos parciales muy pobres ( 3 casos). Es de anotar, por último, que el éxito logracio en la primera serie fue del $83,3 \%$ y en la segunda con un número de casos casi equivalente a la mitad, de $100 \%$.

Resumiendo tenemos: los resultados presentados por el doctor G. Isaza Mejía y colaboradores, sobre aplicación del método sicoprofiláctico en la ciudad de Medellín (Colombia), son de gran interés por considerar que el programa de clases teóricas, salvo algunas anotaciones, ha cumplido a cabalidad con su finalidad; y en lo relativo a la parte práctica, el folleto suministrado a las pacientes y publicado conjuntamente con el informe de resultados, es de un gran valor para la embarazada como guía de entrenamiento; pudiéndosele considerar, con muy ligeras anotaciones, como el tipo ideal. Por último, los datos estadísticos, aunque tomacos sobre un número muy limitado de observaciones, dan cifras a bdas luces excelentes, que deben servir de estímulo para posteriores estudios.

Doctor M. A. Fernández-Bastidas

Calle 63-A, número 10-48. Bogotá, D. E. 